\title{
A NOTE ON THE LOCATION OF CRITICAL POINTS OF POLYNOMIALS
}

\author{
E. B. SAFF AND J. B. TWOMEY
}

AbStract. Let $P(a, 3)$ denote the set of cubic polynomials which have all of their zeros in $|z| \leqq 1$ and at least one zero at $z=a$ $(|a| \leqq 1)$. In this paper we describe a minimal region $D(a, 3)$ with the property that every polynomial in $P(a, 3)$ has at least one critical point in $D(a, 3)$. The location of the zeros of the logarithmic de-

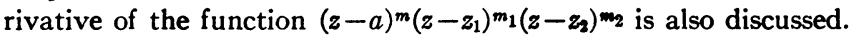

1. Introduction. Let $p(z)$ be a polynomial of degree $n(\geqq 2)$ having all its zeros in the closed disk $\gamma:|z| \leqq 1$. Ilieff has conjectured [1,p. 25 ] that if $a$ is a zero of $p(z)$, then at least one critical point of $p(z)$ (i.e., zero of $p^{\prime}(z)$ ) lies in the disk $|z-a| \leqq 1$. A more difficult problem related to this conjecture is stated in [2] as follows:

Let $\mathcal{P}(a, n)$ be the set of all $n$th degree polynomials which have all of their zeros in $\gamma$ and at least one zero at the point $z=a$. Describe a region $D(a, n)$ such that (i) $D(a, n)$ contains at least one critical point of every $p(z) \in \mathcal{P}(a, n)$ and such that (ii) no proper subset of $D(a, n)$ has property (i).

It is the aim of the present note to describe sets $D(a, 3),|a| \leqq 1$, and thereby improve the results of Schmeisser [3] and others [4], [5] concerning the location of critical points of cubic polynomials. We shall define only those sets $\mathscr{D}(a, 3)$ for which $0 \leqq a \leqq 1$, since the remaining sets can then be obtained by rotation. Our main result is

TheOREM 1. Let $p(z)=(z-a)\left(z-z_{1}\right)\left(z-z_{2}\right)$, where $0 \leqq a \leqq 1,\left|z_{1}\right| \leqq 1$, and $\left|z_{2}\right| \leqq 1$. Let $\Delta(a)$ denote the closed disk

$$
\Delta(a):|z-a / 2| \leqq\left(\left(4-a^{2}\right) / 12\right)^{1 / 2},
$$

and $C(a)$ denote its circumference. Then $p^{\prime}(z)$ has at least one zero in the set $\mathfrak{D}(a, 3)$ defined by

$$
\begin{aligned}
& D(a, 3)=\Delta(a) \backslash[C(a) \cap\{z: \operatorname{Im} z>0\}], \quad a>0, \\
& D(0,3)=\Delta(0) .
\end{aligned}
$$

Presented to the Society, March 28, 1970 under the title Critical points of poly. nomials; received by the editors March 16, 1970.

AMS 1969 subject classifications. Primary 3011.

Key words and phrases. Critical points of polynomials, zeros of the derivative Ilieff conjecture, cubic polynomials, logarithmic derivatives of polynomials. 
Furthermore if $\mathfrak{D}(a, 3)$ is replaced by any proper subset of $\mathscr{D}(a, 3)$, then the assertion is false.

The proof of Theorem 1 is given in $\$ 2$. In $\$ 3$ we consider the location of the zeros of the logarithmic derivative of the function $(z-a)^{m}\left(z-z_{1}\right)^{m_{1}}\left(z-z_{2}\right)^{m_{2}}$, and in $\$ 4$ we mention an open problem that is suggested by our results.

2. Proof of Theorem 1. The proof is based upon the following simple lemma:

LEMMA 1. If $f(\zeta)=b_{2} \zeta^{2}+b_{1} \zeta+b_{0}$ is nonzero in $|\zeta|<1$, then

$$
\left|b_{0}\right|^{2}-\left|b_{2}\right|^{2} \geqq\left|\bar{b}_{1} b_{2}-\bar{b}_{0} b_{1}\right| \text {. }
$$

Proof. The case $b_{2}=0$ is trivial. The case $b_{2} \neq 0$ is at once reduced to $b_{2}=1$.

Let $\alpha, \beta$ be the roots of $\zeta^{2}+b_{1} \zeta+b_{0}=0,|\alpha| \geqq 1,|\beta| \geqq 1$. Then $(|\alpha|-1)(|\beta|-1) \geqq 0$, and so $|\alpha \beta|+1 \geqq|\alpha|+|\beta|$. Multiplying both sides of the last inequality by $|\alpha \beta|-1=\left|b_{0}\right|-1(\geqq 0)$ there follows

$$
\begin{aligned}
\left|b_{0}\right|^{2}-1 & \geqq|\alpha|^{2}|\beta|+|\alpha||\beta|^{2}-|\alpha|-|\beta| \\
& =\left(|\alpha|^{2}-1\right)|\beta|+\left(|\beta|^{2}-1\right)|\alpha| \\
& \geqq\left|\left(|\alpha|^{2}-1\right) \bar{\beta}+\left(|\beta|^{2}-1\right) \bar{\alpha}\right|=\left|b_{1}-b_{0} b_{1}\right|,
\end{aligned}
$$

which proves Lemma 1.

We can now prove

Lемма 2. Let $p(z)=(z-a)\left(z-z_{1}\right)\left(z-z_{2}\right)$, where $0<a \leqq 1,\left|z_{1}\right|=1$, and $\left|z_{2}\right| \leqq 1$. If $p^{\prime}(z)$ has no zero inside $C(a)$, then $z_{2}=\bar{z}_{1}$ or $a=z_{1}=1$ or $a=z_{2}=1$.

Furthermore, if $p_{\alpha}(z)=(z-a)\left(z-e^{i \alpha}\right)\left(z-e^{-i \alpha}\right)$ and

$$
0 \leqq \alpha_{1} \equiv \cos ^{-1}\left(\frac{a+6 A}{4}\right) \leqq \alpha \leqq \cos ^{-1}\left(\frac{a-6 A}{4}\right) \equiv \alpha_{2} \leqq \pi,
$$

where $A \equiv\left(\left(4-a^{2}\right) / 12\right)^{1 / 2}$, then $p_{\alpha}^{\prime}(z)$ has a pair of conjugate zeros and as $\alpha$ varies from $\alpha_{1}$ to $\alpha_{2}$ these zeros describe $C(a)$. If, however, $0 \leqq \alpha<\alpha_{1}$ or $\alpha_{2}<\alpha \leqq \pi$, then $p_{\alpha}^{\prime}(z)$ has a zero inside $C(a)$.

PROOF. Let $z_{1}=\exp \left[i \theta_{1}\right]$ and $z_{2}=r \exp \left[i \theta_{2}\right](0 \leqq r \leqq 1)$. To prove the first part of the lemma we note initially that since $p^{\prime}(z)$ is nonzero inside $C(a)$, the polynomial

$$
P(\zeta) \equiv p^{\prime}(A \zeta+a / 2)=3 A^{2} \zeta^{2}+A\left(a-2 z_{1}-2 z_{2}\right) \zeta+z_{1} z_{2}-a^{2} / 4
$$

is nonzero in $|\zeta|<1$. Hence as a consequence of (1) we have

$$
\left|b_{0}\right|^{2}-\left|b_{2}\right|^{2} \geqq\left|\operatorname{Re}\left(\bar{b}_{1} b_{2}-\bar{b}_{0} b_{1}\right)\right|,
$$


where $b_{k}$ denotes the coefficient of $\zeta^{k}$ in the expansion of $P(\zeta)$. Since

$$
\operatorname{Re}\left(b_{1} b_{2}-\bar{b}_{0} b_{1}\right)=A\left[a\left(1-r \cos \left(\theta_{1}+\theta_{2}\right)\right)-2\left(1-r^{2}\right) \cos \theta_{1}\right]
$$

and $A \geqq a / 2$, we deduce from (2) that

$$
\begin{aligned}
& a^{2}\left(1-r \cos \left(\theta_{1}+\theta_{2}\right)\right) / 2-\left(1-r^{2}\right) \\
& \geqq a^{2}\left(1-r \cos \left(\theta_{1}+\theta_{2}\right)\right) / 2-a \cos \theta_{1}\left(1-r^{2}\right),
\end{aligned}
$$

that is $\left(1-r^{2}\right)\left(a \cos \theta_{1}-1\right) \geqq 0$. Hence either $r=1$ or $1=a=\cos \theta_{1}=z_{1}$. If $r=1$, then (2) implies that

$$
(a / 2-A)\left(1-\cos \left(\theta_{1}+\theta_{2}\right)\right) \geqq 0,
$$

and so either $a / 2=A$, i.e., $a=1$, or $\cos \left(\theta_{1}+\theta_{2}\right)=1$, i.e., $z_{2}=\bar{z}_{1}$. Finally, if $r=1$ and $a=1$, we have equality in (2) and it follows from (1) that

$$
\operatorname{Im}\left(b_{1} b_{2}-b_{0} b_{1}\right)=\sin \left(\theta_{1}+\theta_{2}\right)-\sin \theta_{1}-\sin \theta_{2}=0 .
$$

This last equation implies that $\theta_{1} \equiv 0$ or $\theta_{2} \equiv 0$ or $\theta_{1}+\theta_{2} \equiv 0(\bmod 2 \pi)$, i.e., $z_{1}=1$ or $z_{2}=1$ or $z_{2}=\bar{z}_{1}$. The proof of the first part of Lemma 2 is now complete.

To prove the second part we note that $p_{\alpha}^{\prime}(A \zeta+a / 2)$ has the zeros

$$
\zeta_{1}, \zeta_{2}=\frac{-(a-4 \cos \alpha) \pm\left[(a-4 \cos \alpha)^{2}-36 A^{2}\right]^{1 / 2}}{6 A},
$$

and that the quantity under the radical is nonpositive if $\alpha_{1} \leqq \alpha \leqq \alpha_{2}$. Furthermore, it is easily verified that as $\alpha$ varies from $\alpha_{1}$ to $\alpha_{2}$ the points $\zeta_{1}, \zeta_{2}$ describe the boundary of the unit disk. Finally, if $0 \leqq \alpha<\alpha_{1}$ or $\alpha_{2}<\alpha \leqq \pi$, then $\zeta_{1}$ and $\zeta_{2}$ are real and unequal and, since their product is unity, it follows that one of them lies in $|\zeta|<1$. This implies the second part of Lemma 2 and concludes the proof.

We next establish

Lemma 3. Let $p(z)=(z-a)\left(z-z_{1}\right)\left(z-z_{2}\right)$, where $0 \leqq a \leqq 1,\left|z_{1}\right|<1$, and $\left|z_{2}\right|<1$. Then $p^{\prime}(z)$ has at least one zero inside $C(a)$.

Proof. Since $\left|z_{1}\right|<1$ and $\left|z_{2}\right|<1$, there exists a number $\rho(0<\rho<1)$ such that

$$
q(w) \equiv p(\rho w+(1-\rho) a)=\rho^{3}(w-a)\left(w-w_{1}\right)\left(w-w_{2}\right),
$$

where $\left|w_{1}\right|=1$ and $\left|w_{2}\right| \leqq 1$. By Lemma $2, q^{\prime}(w)$ has a zero in $|w-a / 2| \leqq A$ and hence $p^{\prime}(z)$ has a zero $(\neq 1)$ in the disk

$$
\gamma(\rho, a):|z-a / 2-(1-\rho) a / 2| \leqq \rho A .
$$

Since $A \geqq a / 2$ with equality only for $a=1$, it is readily verified that 
every point of $\gamma(\rho, a)$, except $z=1$ in the case $a=1$, lies inside $C(a)$. This proves the lemma.

Combining Lemmas 2 and 3 we clearly obtain the first part of Theorem 1 for $a>0$. For $a=0$ the required result is an immediate consequence of the fact that the modulus of the product of the zeros of $p^{\prime}(z)$ is less than or equal to $1 / 3$.

To prove the second part of Theorem 1 for the case $a>0$ it suffices to show that for any point $z_{0}$ inside $C(a)$ there exists $p(z) \in \mathcal{P}(a, 3)$ such that $p^{\prime}\left(z_{0}\right)=0$ and $p^{\prime}(z) \neq 0$ for all $z\left(\neq z_{0}\right)$ in $D(a, 3)$. Consider first the polynomial

$$
p(z, t)=(z-a)\left(z-e^{i \theta}\right)(z-\beta(t)),
$$

where $\beta(t)=(1-t) a+t e^{i \theta} \quad(0 \leqq t \leqq 1)$. For $t=0$ we have $p(z, 0)$ $=(z-a)^{2}\left(z-e^{i \theta}\right)$ so that $p^{\prime}(z, 0)$ has a zero at $a$ and at $\left(a+2 e^{i \theta}\right) / 3$. It is easy to see that the second zero lies outside $C(a)$ except for the trivial case $a=e^{i \theta}=1$. As $t$ varies continuously from 0 to 1 , each root of $p^{\prime}(z, t)$ varies continuously on the line segment $\left[a, e^{i \theta}\right]$, one from $a$ to $\left(2 a+e^{i \theta}\right) / 3$, the other from $\left(a+2 e^{i \theta}\right) / 3$ to $e^{i \theta}$ (compare [6, p. 24]). It follows, therefore, that no point $z_{0}$ of the closed disk $|z-2 a / 3|$ $\leqq 1 / 3$ can be omitted from $D(a, 3)$.

Suppose next that $z_{0}$ is a point inside $C(a)$ for which $\left|z_{0}-2 a / 3\right|$ $>1 / 3$, and choose $z_{0}^{\prime}\left(\left|z_{0}^{\prime}\right|>1\right)$ such that the derivative of $P_{0}(z)$ $\equiv(z-a)\left(z-z_{0}^{\prime}\right)^{2}$ has a zero at $z_{0}$. We note that

$$
\frac{P_{0}^{\prime}\left(z_{0}\right)}{P_{0}\left(z_{0}\right)}=\frac{1}{z_{0}-a}+\frac{2}{z_{0}-z_{0}^{\prime}}=0 \text {. }
$$

Now consider the mapping $l(z) \equiv 1 /\left(z_{0}-z\right)$. Under $w=l(z)$ the unit circumference is mapped onto a circle $\Gamma$ and $z_{0}^{\prime}$ is mapped to a point $w_{0}^{\prime}$ inside $\Gamma$. Choose $w_{1}^{\prime}, w_{2}^{\prime}$ on $\Gamma$ such that $w_{0}^{\prime}=\left(w_{1}^{\prime}+w_{2}^{\prime}\right) / 2$, and let $z_{1}^{\prime}, z_{2}^{\prime}$ be the points on $|z|=1$ that satisfy $w_{1}^{\prime}=l\left(z_{1}^{\prime}\right), w_{2}^{\prime}=l\left(z_{2}^{\prime}\right)$. It follows that

$$
\frac{2}{z_{0}-z_{0}^{\prime}}=\frac{1}{z_{0}-z_{1}^{\prime}}+\frac{1}{z_{0}-z_{2}^{\prime}}
$$

and, combining (3) and (4), we deduce that the derivative of $p_{0}(z)$ $\equiv(z-a)\left(z-z_{1}^{\prime}\right)\left(z-z_{2}^{\prime}\right)$ has a zero at $z=z_{0}$. Since $\left|z_{1}^{\prime}\right|=\left|z_{2}^{\prime}\right|=1$ implies that $\left|z_{1}^{\prime} z_{2}^{\prime}-a^{2} / 4\right| \geqq 3 A^{2}$, it is easily seen by considering the polynomial $p_{0}^{\prime}(A \zeta+a / 2)$ that the second zero of $p_{0}^{\prime}(z)$ lies outside $C(a)$. This proves Theorem 1 for $a>0$.

For the case $a=0$ we observe that the derivative of

$$
z\left(z-r e^{i \alpha}\right)\left(z-r e^{i(\alpha+\pi / 3}\right), \quad 0 \leqq \alpha \leqq 2 \pi, \quad 0 \leqq r \leqq 1,
$$


has a double zero at $z=r e^{i(\alpha+\pi / 6)} / \sqrt{ } 3$, and hence no point may be omitted from $D(0,3)$. This completes the proof of Theorem 1 .

3. The logarithmic derivative. By applying the methods of $\$ 2$ it is possible to prove

THEOREM 2. Let $m, m_{1}$, and $m_{2}$ be nonnegative real numbers with $n \equiv m+m_{1}+m_{2}>0$, and suppose

$$
F(z)=\frac{m}{z-a}+\frac{m_{1}}{z-z_{1}}+\frac{m_{2}}{z-z},
$$

where $0 \leqq a \leqq 1,\left|z_{1}\right| \leqq 1$, and $\left|z_{2}\right| \leqq 1$. Set

$$
\alpha(m, n) \equiv(n-m) a /(n+m), \quad A(m, n) \equiv\left(m\left(1-\alpha(m, n)^{2}\right) / n\right)^{1 / 2} .
$$

Then $F(z)$ has at least one zero in the disk

$$
|z-\alpha(m, n)| \leqq A(m, n) .
$$

We note that if $p(z)$ is a polynomial of the form

$$
p(z)=(z-a)^{m}\left(z-z_{1}\right)^{m_{1}}\left(z-z_{2}\right)^{m_{2}},
$$

where $0 \leqq a \leqq 1,\left|z_{1}\right| \leqq 1$, and $\left|z_{2}\right| \leqq 1$, then Theorem 2 implies that $p(z)$ has at least one critical point distinct from $a$ in the disk (5). It follows easily from this that Ilieff's conjecture is true for such $p(z)$.

4. Conjecture. The equation

$$
F(z)=\frac{m}{z-a}+\sum_{k=1}^{s} \frac{m_{k}}{z-z_{k}}=0
$$

$\left(m \geqq 0, m_{k} \geqq 0, n=m+m_{1}+\cdots+m_{s}>0,0 \leqq a \leqq 1,\left|z_{k}\right| \leqq 1(1 \leqq k \leqq s)\right)$ has a root $\zeta$ satisfying

$$
|\zeta-\alpha| \leqq\left\{(1-\alpha)^{--1}\left(\frac{m}{n}+\frac{\alpha(s-1) m}{n}\right)\right\}^{1 / 8}
$$

where $\alpha=(n-m) a /(n+(s-1) m)$.

Support for the conjecture comes from Theorem 2 (the case $s=2$ ). It is also easy to verify that the conjecture is true for $a=0$. Its truth for $a=1$ follows from a modification of the proof in [2].

We remark that for the case where $m=1, m_{k}=1(1 \leqq k \leqq s)$, the conjecture is due to J. S. Ratti and is sharper than Ilieff's.

The authors wish to thank the referee for suggesting an elementary proof of Lemma 1. 


\section{REFERENCES}

1. W. K. Hayman, Research problems in function theory, Athlone Press, London, 1967. MR 36 \#359.

2. A. W. Goodman, Q. I. Rahman and J. S. Ratti, On the zeros of a polynomial and its derivative, Proc. Amer. Math. Soc. 21 (1969), 273-274. MR 39 \#421.

3. G. Schmeisser, Bermerkungen zu einer Vermutung von Ilieff, Math. Z. 111 (1969), 121-125.

4. D. A. Brannan, On a conjecture of Ilieff, Proc. Cambridge Philos. Soc. 64 (1968), 83-85. MR 36 \#3958.

5. A. Joyal, On the zeros of a polynomial and its derivative, J. Math. Anal. Appl. 26 (1969), 315-317. MR 39 \#420.

6. J. L. Walsh, The location of critical points of analytical and harmonic functions, Amer. Math. Soc. Colloq. Publ., vol. 34, Amer. Math. Soc., Providence, R. I., 1950. MR 12, 249.

University of South Florida, Tampa, Florida 33620 